\title{
Maltrato en adultos mayores en Saltillo Coahuila
}

\section{Elder abuse in Saltillo Coahuila}

\author{
LÓPEZ-NIEBLA, Rosa María†*, MARTÍNEZ-CÁRDENAS, Juana María, TERRAZAS-MEDINA, \\ Tamara Isabel y PÉREZ-CASTRO, Francisco Isaí
}

Universidad Autónoma de Coahuila, Instituto de Enseñanza Abierta, Unidad Saltillo.

ID $1^{\text {er }}$ Autor: Rosa María, López-Niebla / ORC ID: 0000-0001-7260-3300, arXiv Author: 8GQUZR-P8NFDZ, CVU CONACYT ID: 953192

ID $1^{\text {er }}$ Coautor: Juana María, Martínez-Cárdenas / ORC ID: 0000-0003-1004-9652 / Researcher ID Thomson: X-23702018, CVU CONACYT ID: 949979

ID $2^{\text {do }}$ Coautor: Tamara Isabel, Terrazas-Medina / ORC ID 0000-0002-6581-190X, arXiv Author ID: 8GQUZR-P8NFDZ y CVU CONCAYT ID: 929839

ID $3^{\text {er }}$ Coautor: Francisco Isaí, Pérez-Castro / ORC ID: 0000-0002-6779-7881, arXiv Author ID: 1908.09397, CVU CONACYT ID: 929950

DOI: $10.35429 / J T M S .2019 .14 .5 .29 .34$

Recibido 11 de Enero 2019; Aceptado 30 Marzo, 2019

\section{Resumen}

El presente trabajo se realizó por maestros de la Universidad Autónoma de Coahuila, su objetivo fue diseñar un instrumento para evaluar el maltrato a personas de la tercera edad en la ciudad de Saltillo, Coahuila, para implementar con los resultados obtenidos, programas de servicio social que brinden información sobre la violencia en la tercera edad, identificar comportamientos del agresor, observador y la víctima, así como conocer sus derechos fundamentales e instituciones a dónde acudir. El instrumento se aplicó en el Hospital Geriátrico, participaron 35 adultos mayores, con edades entre 60 y 90 años. Se inspiró en la Validación Psicométrica del cuestionario "Así nos llevamos en la escuela", y el Cuestionario elaborado por INAPAM Y SEDESOL, de ambos instrumentos resultó la adaptación que consta de 35 reactivos, dispersos en 4 factores: físico, psicológico, daño a la propiedad y financiero y hostigamiento sexual. Los resultados muestran que existe maltrato en este colectivo, de $19 \%$, aunque no es muy marcado su manifestación, si lo hay, corroborando lo que dicen otros estudios de que este problema de salud es callado, por la persona de la tercera edad por lealtad a valores familiares o por temor a represalias de la persona de quien lo cuida.

Maltrato, Adulto mayor, Tipos de violencia

\begin{abstract}
The present work was carried out by teachers of the Universidad Autonoma de Coahuila, its objective was to design an instrument to evaluate the abuse of the elderly in the city of Saltillo, Coahuila, to implement with the results obtained, social service programs that provide information on violence in the elderly, identify behaviors of the aggressor, observer and victim, as well as know their fundamental rights and institutions to go to. The instrument was applied at the Geriatric Hospital, 35 older adults participated, aged between 60 and 90 years. It was inspired by the Psychometric Validation of the questionnaire "This is how we get along in school", and the Questionnaire prepared by INAPAM and SEDESOL, of both instruments resulted in the adaptation consisting of 35 reagents, dispersed in 4 factors: physical, psychological, damage to the property and financial, and sexual harassment. The results show that there is abuse in this group, of 19\%, although its manifestation is not very marked, if there is one, corroborating what other studies say that this health problem is silent, by the person of the third age by loyalty to family values or for fear of reprisals from the person who takes care of him.
\end{abstract}

Abuse, Elderly, Types of violence

Citación: LÓPEZ-NIEBLA, Rosa María, MARTÍNEZ-CÁRDENAS, Juana María, TERRAZAS-MEDINA, Tamara Isabel y PÉREZ-CASTRO, Francisco Isaí. Maltrato en adultos mayores en Saltillo Coahuila. Revista Transdisciplinaria de Estudios Migratorios. 2019, 5-14: 29-34

\footnotetext{
*Correspondencia al Autor (correo electrónico: rosy_lopez_niebla@ hotmail.com)

$\uparrow$ Investigador contribuyendo como primer autor.
} 


\section{Introducción}

Cada vez, se hacen presente en nuestra sociedad, un cambio constante en los estilos de vida, en los conceptos, valores familiares y tipos de familia, así como también la vorágine de actividades fuera del hogar que abruma a los miembros de la familia y los avances de la medicina, el aumento de la esperanza y calidad de vida ha originado un numeroso colectivo de personas de la tercera edad.

Lejos de representar una fuente de sabiduría y respeto, como era el status que los ancianos guardaban en ancestrales sociedades, hoy, constituyen "un estorbo" para las familias que los atienden. Esta concepción de poco respeto y valoración a la edad del anciano, conlleva a una tipificación de violencia denominada "Maltrato a personas de la tercera edad"

Cada vez, nuestra sociedad presenta cambios constantes que van desde los estilos de vida caracterizados hoy día por su "aceleramiento" en pos del logro de la satisfacción de necesidades posmodernas desde la adquisición de un teléfono móvil de tecnología de punta, hasta una lista inimaginable de necesidades impuestas por la mercadotecnia y la tecnología, que como bumerang repercute en todos los subsistemas de la sociedad, principalmente el agobio, se asienta en lo económico, tal y como se observa en nuestro país con deplorables salarios, desempleo, falta de oportunidades de trabajo que conllevan a la pobreza y al rubro insuficiente y de alto costo de la vivienda, sobre todo las adquiridas mediante instituciones gubernamentales construidas hasta en $82 \mathrm{~m}^{2}$ de superficie cuyo espacio no cumple con las condiciones adecuadas para favorecer la convivencia familiar.

Otros cambios que se manifiestan en la familia actual, son la jerarquización de valores familiares, proyectos de vida personales, las actividades fuera del hogar de índole escolar, profesional, laboral, resulta abrumador para los miembros de la familia brindar atención a las personas de la tercera edad que viven con hijos, nietos, hermanos, toda vez que no se dispone ni de tiempo, ni solvencia económica, ni espacio físico para brindar una calidad de vida digna a los ancianos de la familia, convirtiéndose este colectivo en un grupo vulnerable y en un problema de salud.
Por todo lo anterior, el Instituto de Enseñanza Abierta, US de la Universidad Autónoma de Coahuila, se inspira en realizar una investigación para determinar si existe o no maltrato en las personas de la tercera edad y de qué tipo es su prevalencia.

Como resultado de campañas mundiales y nacionales sobre el control de la natalidad, durante la segunda mitad del siglo pasado, se observó un notable decremento en nacimientos y en mortalidad, en tanto que un considerable aumento de la esperanza de vida en la población adulta; lo que ha incidido en un proceso de envejecimiento a nivel global. Para contribuir a tomar conciencia de este hecho, la Organización de las Naciones Unidas declaró 1999 como el Año Internacional de las Personas de Edad y a partir de esta fecha proliferó la atención a este grupo desde diferentes rubros.

En nuestro país, no es ajeno a este proceso de envejecimiento. Reporta el INEGI, que en 2014, la base de la pirámide poblacional es más angosta que en 1990 debido a que la proporción de niños y jóvenes es menor, observándose un ensanchamiento en la cúspide en virtud del aumento de los adultos mayores que pasó en este período de 6.2 a $9.7 \%$ y se espera que en 2050 se incremente a 21.5 por ciento, lo cual significa que el número de adultos mayores se duplicó en las últimas décadas, pasando de 5 a 11.7 millones de 1990 a 2014. (CONAPO).

La palabra maltrato, tiene una connotación fuerte en todas las relaciones humanas, escucharla, sorprende, provoca repudio y asusta, porque revela falta de agradecimiento a una persona vulnerable que en su edad productiva aportó con su trabajo a la construcción del país, de su familia, de su comunidad.

$\mathrm{Al}$ respecto, (Bennett, Kingston y Penhale, 1997: 1). Dice que "El maltrato a las personas mayores es el último capítulo de la violencia familiar y por lo tanto bastante desconocido en la actualidad. Por su parte, T. Bazo pone de manifiesto que el problema de los malos tratos a las personas ancianas existe en todas las sociedades, ya que "la violencia en general y la violencia familiar en particular se producen como resultado de la capacidad de los seres humanos para producir daño a otros, sea de forma voluntaria o involuntaria". 
El maltrato a personas de la tercera edad, ha generado numerosos trabajos e investigaciones realizados en las últimas décadas, cuyos resultados indican la magnitud del problema social y de salud que representa para nuestra sociedad,

Este tema, por su relevancia y por ser prioritario conocerlo, detectarlo y elaborar planteamientos sólidos que orienten propuestas de cambio en las políticas públicas y nuevas leyes, es la fuente de inspiración para la elaboración de un instrumento para evaluar el maltrato en personas de la tercera edad en Saltillo, Coahuila.

Para ello, se hace necesario, definir ¿qué es el maltrato de los ancianos?

\section{Metodología a desarrollar}

La presente investigación se llevó a cabo en el Hospital Geriátrico de la Ciudad de Saltillo Coahuila. Se aplicaron 35 encuestas a adultos mayores en edades entre 60 y 90 años. La investigación es de tipo descriptiva y cuyas interrogantes son:

¿Existe maltrato en personas de la tercera edad en la Ciudad de Saltillo, Coahuila?

¿Cuál es el tipo de maltrato que prevalece?

Se utilizó un muestreo no probabilístico y por accidente, la muestra se conformó por 35 sujetos. El instrumento de recolección de datos utilizado se diseñó para tal efecto. Las variables se distribuyen en cuatro factores que son: Maltrato Físico con 10 variables; Maltrato Psicológico con 10 variables; Disposición de pertenencias y Finanzas (abuso a la propiedad y dinero, para otros autores) 8 variables y Acoso Sexual 5 variables.

\section{Objetivo General}

Elaborar un instrumento que evalúe el maltrato a personas de la Tercera edad en Saltillo, Coahuila a fin de conocer si existe el maltrato en personas de la tercera edad en Saltillo, Coahuila.

\section{Objetivos particulares}

Detectar el maltrato en personas de la Tercera edad en la Ciudad de Saltillo, Coahuila.
Identificar tipos de maltrato de mayor incidencia en personas de la tercera edad de Saltillo, Coahuila

\section{Diseño}

Guba (1985) en su artículo "Criterios de credibilidad en la investigación naturalista" señala que el Diseño es abierto, no estructurado, que se va desarrollando a medida que evoluciona la investigación, en cuanto al escenario, este mismo autor lo señala como el lugar donde se lleva a cabo la investigación en un contexto natural, en el lugar donde ocurren los hechos.

En un estudio descriptivo se seleccionan una serie de conceptos o variables y se mide cada una de ellas independientemente de las otras, con el fin, precisamente, de describirlas. Estos estudios buscan especificar las propiedades importantes de personas, grupos, comunidades o cualquier otro fenómeno.

Por lo que para efectos de este trabajo, se utilizó la metodología de la investigación cualitativa, descriptiva referida por Taylor y Bogdan ( 1986:20) "como aquella que produce datos descriptivos: de las personas habladas o escritas y la conducta observable", se consideró este método descriptivo por considerarlo apropiado de acuerdo al objetivo de la investigación y al problema de éste para detectar e identificar cuál es el tipo de maltrato del que son objeto los adultos mayores de la Ciudad de Saltillo, Coahuila. Con dicha descripción se intenta ir construyendo un concepto o noción fundamentada acerca del fenómeno del maltrato y el sujeto de la tercera edad.

Este tipo de estudio selecciona una serie de cuestiones y se mide cada una de ellas independientemente, para así describir lo que se investiga." (Hernández, 1998: 60). El diseño de investigación es No-Experimental o Ex postfacto, puesto que "se realiza sin manipular deliberadamente variables. Lo que se hizo en la investigación fue observar fenómenos tal y cómo se dan en su contexto natural, para después analizarlos (Kerlinger, op.cit. Hernández, 1998), tampoco se asignó aleatoriamente a los sujetos o las condiciones (Hernández et al, 1998: 184). Esta investigación nos permitió observar el fenómeno del maltrato tal cual como se presenta en la realidad, como un hecho ya sucedido, al igual que sus efectos. 
La Unidad de Análisis son los adultos mayores maltratados que acuden al Hospital Geriátrico de la Ciudad de Saltillo, Coahuila. El instrumento: mide 33 conductas de representación de maltrato, dispersas en 4 factores a saber:

$\begin{array}{lll}\text { MF } & \text { Factor Físico } & 10 \text { variables. } \\ \text { MP } & \text { Factor Psicológico } & 10 \text { variables } \\ \text { DDPF } & \text { Disposición de propiedades } & 8 \text { variables } \\ & \text { y finanzas } & \\ \text { AS } & \text { Abuso sexual } & 5 \text { variables } \\ & \text { Total } & 33 \text { variables }\end{array}$

Los 5 tipos de maltrato a las personas de la Tercera edad, (J. Lowick R, 1999) se describen como:

Maltrato físico: toda forma de castigo corporal. Se manifiesta mediante el golpe o daño físico, tales como pellizcos, jalones de pelo o cualquier lesión en el cuerpo.

Abuso sexual: al realizarse un acto de naturaleza sexual contra la voluntad de la persona adulta mayor.

\section{Maltrato}

\section{emocional}

psicológico: comprende desde insultos, burlas, rechazo, desvalorización y/o ausencia de expresiones positivas hacia el adulto mayor. También se le llama maltrato por negligencia, se producen cuando no se atienden las necesidades de alimentación, salud y afecto que las personas adultas mayores requieren y se merecen.

Disposición de propiedades y finanzas: Cuando hablamos de abuso patrimonial o maltrato económico nos referimos al "mal uso o explotación de dinero o de las posesiones del anciano, así como el bloqueo de acceso a éste de dichos bienes" (Ruipérez y Llorente, 1996), citado por Lamas sf lo que implica " daño, pérdida, transformación, sustracción, destrucción, retención o falsificación de objetos, instrumentos de trabajo, documentos personales, bienes, valores, derechos o recursos económicos destinados a satisfacer las necesidades de las personas mayores" (Jimenez y Román, 1996). Citado por Lamas sf

\section{Resultados}

Los datos que arrojó la presente investigación son como lo describen las siguientes gráficas.

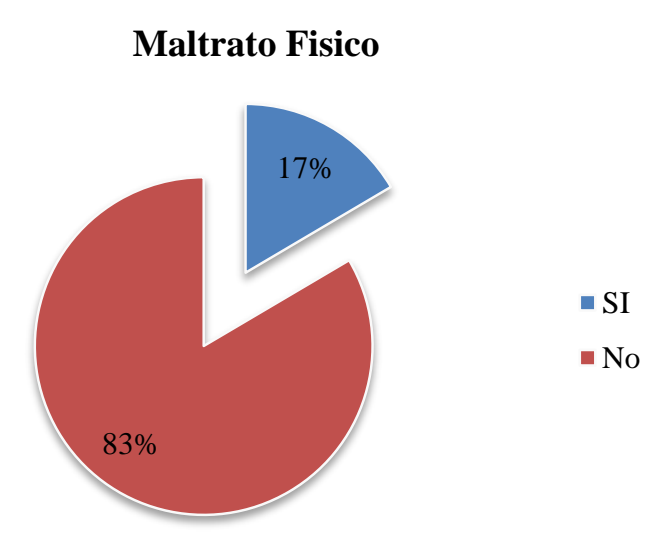

Gráfica 1 Maltrato Físico

Se puede ver en la gráfica 1 que existe un $17 \%$ de probabilidad de maltrato físico, en cambio el $83 \%$ dice que no fue maltratado, los aplicadores del instrumento comunicaron que los encuestados a veces dudaban en su respuesta o tardaban en contestar.

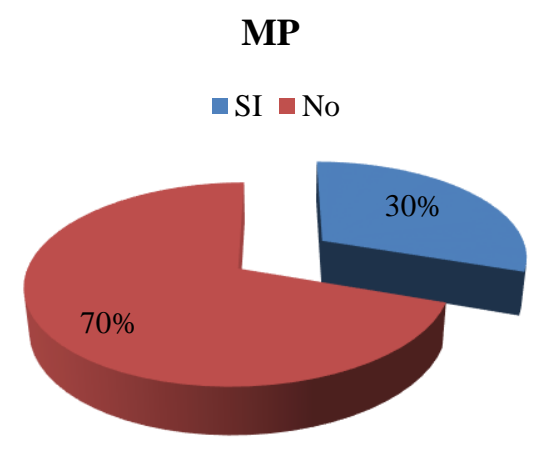

Gráfica 2 Maltrato Psicológico

En la gráfica 2 se muestra que existe una mayor probabilidad con un $30 \%$ de maltrato psicológico.

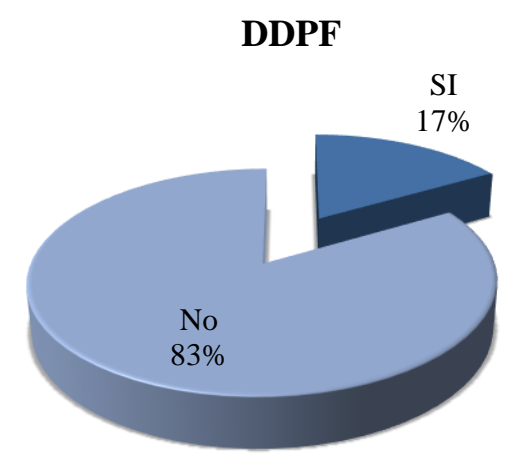

Gráfica 3 Disposición de propiedades y finanzas

Como se observa en la gráfica 3 que existe un $17 \%$ de probabilidad en la disposición de propiedades y finanzas. 


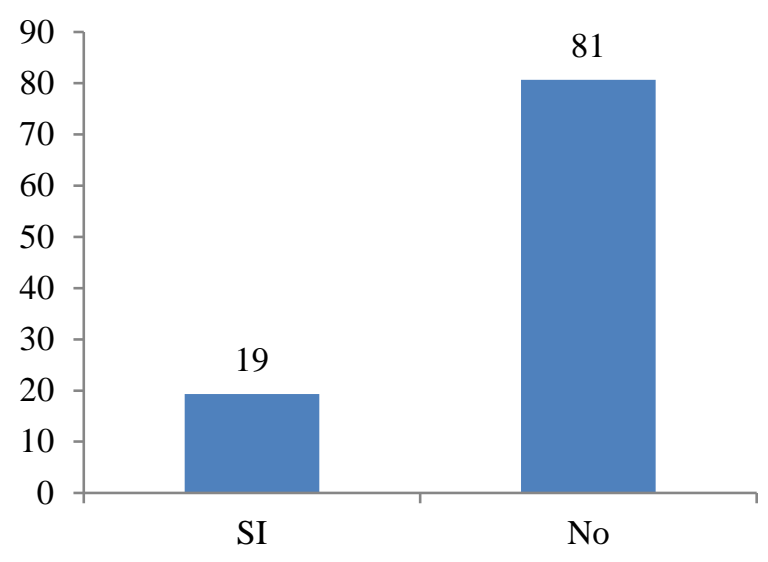

Gráfica 4 Concentrado total

En la gráfica 4 se muestra la suma total respecto a las encuetas, este es el concentrado de todos los tipos de maltrato y por lo que se observa, los sujetos manifestaron que reciben maltrato un $19 \%$.

SUMA TOTAL
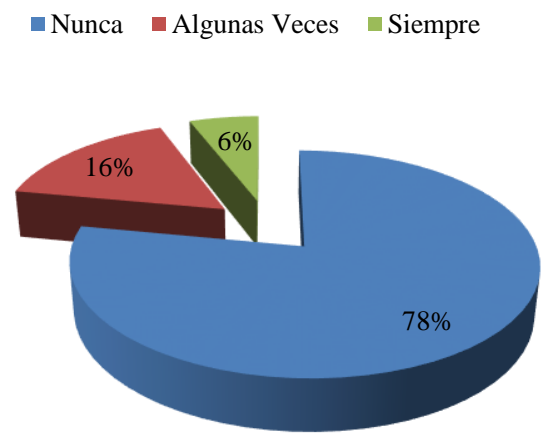

Gráfica 5 Frecuencia del maltrato

En cuanto a la frecuencia con que se presenta el maltrato los resultados arrojan que el $78 \%$ nunca recibe maltrato, el $16 \%$ algunas veces y el $6 \%$ siempre lo recibe.

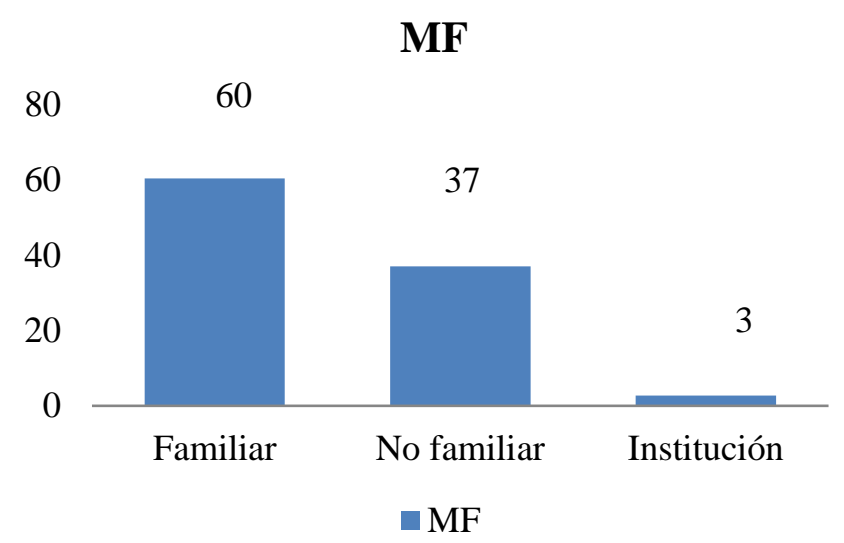

Gráfica 6 Persona que incurre en el maltrato físico
Como se muestra en la gráfica 6, en el hogar, la familia es la que más abusa en el maltrato físico, con un $60 \%$ de incidencia.

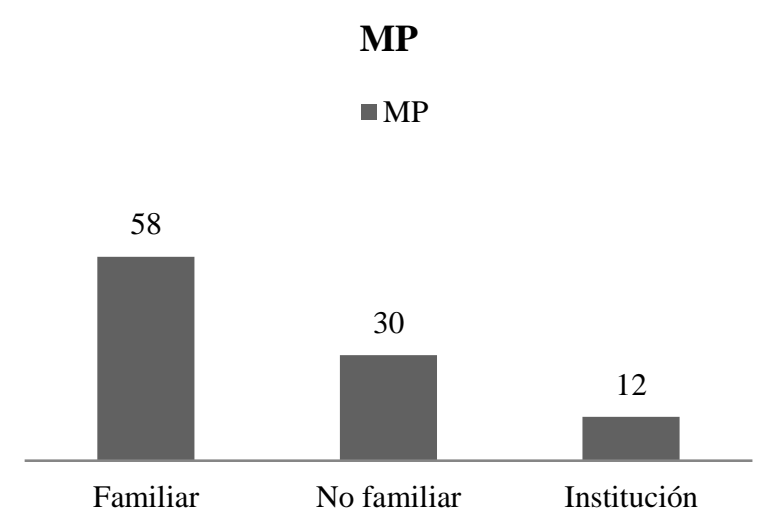

Gráfica 7 Maltrato Psicológico

Como se puede observar en la gráfica 7 , la familia es la que más abusa psicológicamente, como ocurre en el maltrato físico.

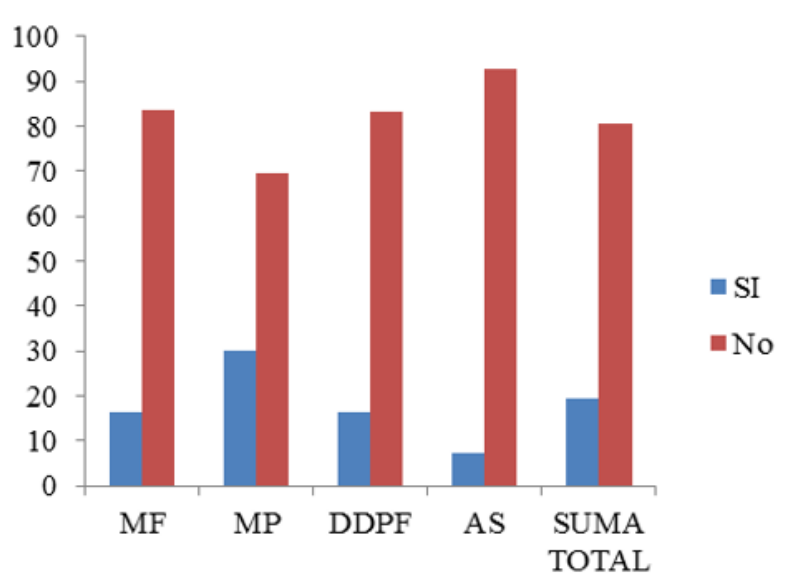

Gráfica 8 Maltrato

En cuanto al maltrato, como se puede observar en la gráfica 8 , menos del $30 \%$ de los encuestados manifiestan que sufren de cualquiera de los cinco tipos de maltrato.

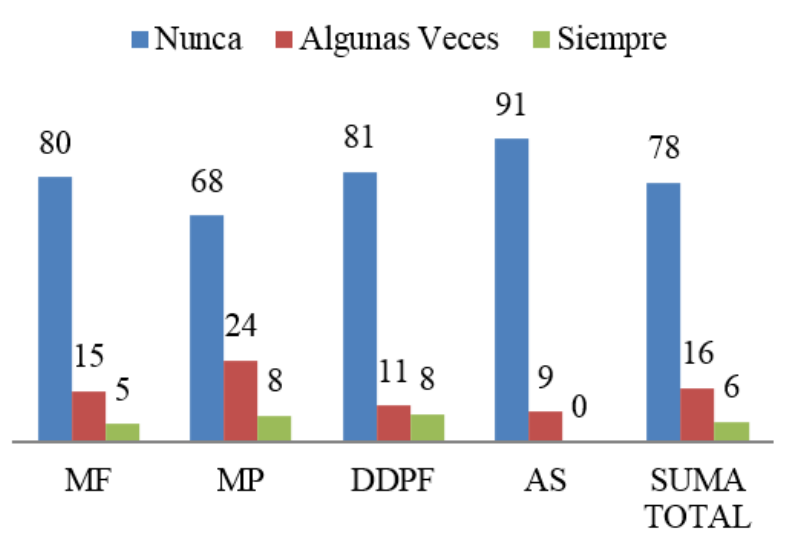

Gráfica 9 Frecuencia con que se sufre el maltrato 
Los sujetos manifiestan que la frecuencia con que sufren el maltrato, cualquiera de sus tipos, es de menos del $24 \%$.

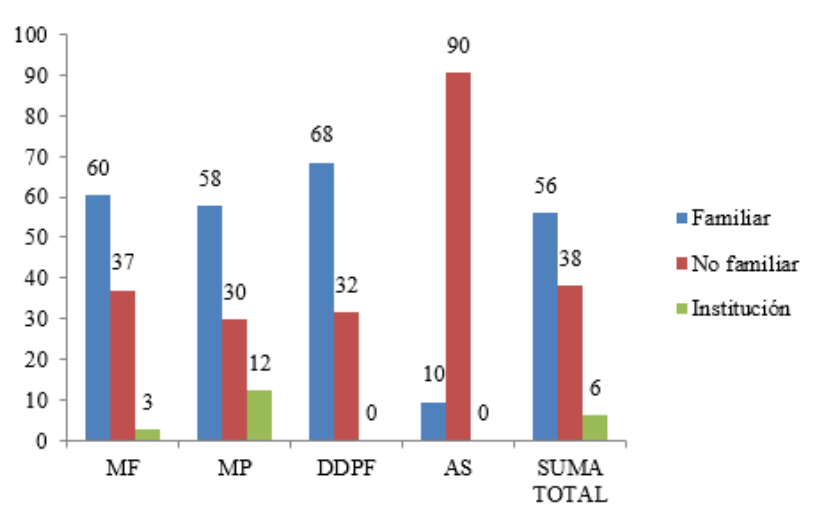

Gráfica 10 Persona que propicia el maltrato

Se muestra en la gráfica 10 , que cuando se sufre maltrato, en cualquiera de sus tipos, la mayor parte proviene de un familiar

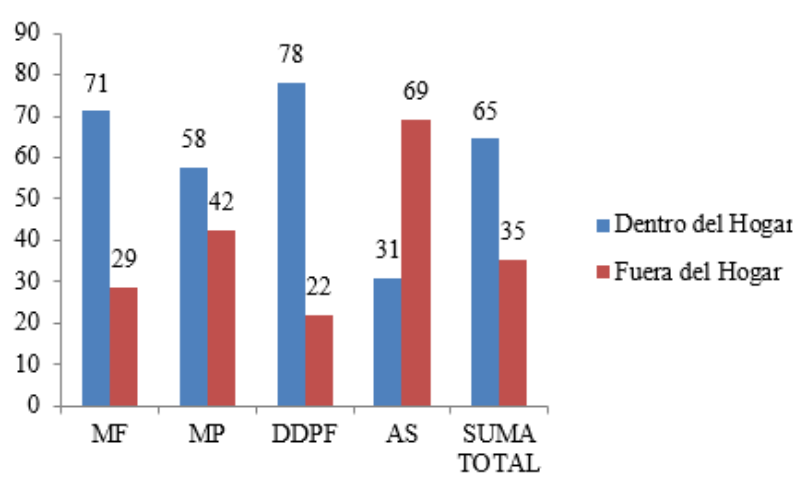

Gráfica 11 Lugar en el que ocurre el maltrato

Como se puede observar en la gráfica 11 , el hogar es donde se manifiesta el maltrato, en cualquiera de sus cinco tipos.

\section{Conclusiones}

De acuerdo con los datos arrojados por el instrumento para evaluar si existe o no y el tipo que prevalece en personas de la tercera edad de Saltillo Coahuila, nos orienta a concluir que existe maltrato en este colectivo, de un 19\%, aunque no es muy marcado su manifestación, si lo hay, corroborando lo que dicen otros estudios de que este problema de salud es callado, por la persona de la tercera edad por lealtad a valores familiares o por temor a represalias de la persona de quien depende para su cuidado. Dentro de este grado de maltrato prevalecen con mayor frecuencia el maltrato psicológico, lo cual es comprensible, toda vez que el cansancio o el perfil psicológico del cuidador lo provoca.
Otro dato relevante está manifestado en el rubro donde se infringe el maltrato, y en este instrumento revela que se da con más incidencia dentro de la familia. Éste dato nos sugiere pensar que es viable la respuesta prevalente del maltrato en despojo de propiedad y finanzas, por la vulnerabilidad de la persona de la tercera edad al depender del cuidado de su familia.

\section{Referencias}

Abuso o maltrato en el Adulto mayor. Recuperado de http://escuela.med.puc.cl/publ/manualgeriatria/ PDF/AbusoMaltrato.pdf Consultado el 5 de noviembre del 2015.

Baeza Ulloa, Verónica Del Carmen \& Poblete Berrios, Rosa Carolina. (2006) Adulto Mayor y maltrato. Universidad Academia de Humanismo Cristiano. Chile, Santiago. Recuperado de http://bibliotecadigital.academia.cl/bitstream/ha ndle/123456789/453/tesis\%20ttraso155.pdf?seq uence $=4 \&$ isAllowed $=y \quad$ Consultado el 5 de noviembre del 2015.

INEGI. XI Censo General de Población y Vivienda. (2014, 25 de septiembre). "ESTADÍSTICAS A PROPÓSITO DEL... DÍA INTERNACIONAL DE LAS PERSONAS DE EDAD”. Recuperado de http://www.inegi.org.mx/saladeprensa/aproposit o/2014/adultos0.pdf. Consultado el 5 de noviembre del 2015.

Lamas, H. \& Lamas, Fco. (2012) Aula Virtual ALPJF El maltrato al adulto mayor. Recuperado de http://psicologiajuridica.org/archives/2831 Consultado el 2 de noviembre del 2015.

Universidad Nacional Abierta y a Distancia. Investigación Exploratoria, Descriptiva, Correlacional y Explicativa. Recuperado de http://datateca.unad.edu.co/contenidos/100104/ 100104_EXE/leccin_6_investigacin_explorato ria_descriptiva_correlacional_y_explicativa.ht ml Consultado el 10 de noviembre del 2015. 